\title{
Dynamic modeling of the reactive twin-screw co-rotating extrusion process: experimental validation by using inlet glass fibers injection response and application to polymers degassing
}

\begin{tabular}{|r|l|}
\hline Journal: & Industrial \& Engineering Chemistry Research \\
\hline Manuscript ID: & ie-2012-00698k.R1 \\
\hline Manuscript Type: & Article \\
\hline Date Submitted by the Author: & 20 -Jul-2012 \\
\hline Complete List of Authors: & $\begin{array}{l}\text { Bilongo, Tchicaya; Université de Lyon, Université Lyon 1, Laboratoire } \\
\text { d'Automatique et de Génie des Procédés, UMR CNRS 5007 } \\
\text { Couenne, Françoise; CNRS, Laboratoire d'Automatique et de Génie des } \\
\text { Procédés, UMR CNRS 5007 } \\
\text { Jallut, Christian; Université de Lyon, Université Lyon 1, Laboratoire } \\
\text { d'Automatique et de Génie des Procédés, UMR CNRS 5007 } \\
\text { Le Gorrec, Yann; ENSMM, FEMTO-ST, UMR CNRS 6174 } \\
\text { Di Martino, Audrey; Rhodia, Centre de Recherches et de Technologies de } \\
\text { Lyon-CRTL }\end{array}$ \\
\hline
\end{tabular}




\title{
Dynamic modeling of the reactive twin-screw co-rotating extrusion process: experimental validation by using inlet glass fibers injection response and application to polymers degassing
}

\author{
T. Goma-Bilongo ${ }^{1}$, F. Couenne ${ }^{1}$, C. Jallut ${ }^{1 *}$, Y. Le Gorrec ${ }^{2}$, A. Di Martino ${ }^{3}$ \\ ${ }^{1}$ Université de Lyon, F-69622, Lyon, France; \\ Université Lyon 1, Villeurbanne, LAGEP, UMR CNRS 5007, \\ CPE Lyon, 43 bd du 11 novembre 1918, 69622 Villeurbanne Cedex, France. \\ ${ }^{2}$ ENSMM Besançon, FEMTO-ST/AS2M, UMR CNRS 6174, 24 rue Alain Savary, 25000 \\ Besançon, France. \\ ${ }^{3}$ Rhodia, Centre de Recherches et de Technologies de Lyon-CRTL, \\ 85 Rue des Frères Perret - BP 62, 69192 Saint-Fons Cedex, France.
}

*Corresponding author: jallut@lagep.univ-lyon1.fr

Abstract: In this paper is described an original dynamic model of a reactive co-rotating twinscrew extrusion (TSE) process operated by the Rhodia company for the Nylon-66 degassing finishing step. In order to validate the model, dynamic experiments have been performed on a small-scale pilot plant. These experiments consist in a temporary injection of glass fibers at the inlet of the extruder after it has reached a given operating point. The outlet glass fibers mass fraction time variation is then measured. This experiment does not lead to the RTD measurement. As a matter of fact, due to the high quantity of glass fibers that is introduced, the behavior of the flow through the extruder is perturbed so that the glass fibers cannot be considered as an inert tracer. The dynamic model that we have published elsewhere (Choulak et al., Ind. Eng. Chem. Res., 2004, 43(23), 7373-7382) is adapted to take into account this nonlinear behavior of the extruder with respect to the glass fibers injection and is favorably compared to experimental results. The description of the degassing operation is also included in the model. The model allows simulations of the complete dynamic behavior of the process. When the steady state is reached, the good position of the degassing vent with respect to the partially and fully filled zones positions can also be checked, thus illustrating the way the model can be used for design purposes.

Keywords: reactive extrusion, degassing, dynamic modeling, tracer injection

\section{Introduction}

Extrusion processes are used in the chemical industries as chemical reactors for polymer synthesis and modification. ${ }^{1}$ The co-rotating twin-screw extruder is of particular interest due to its modular geometry. Another interesting property is that the filling ratio can be less then one in some part of the system according to the screw configuration and the operating conditions. $^{2}$ This property allows introducing or removing chemical species. ${ }^{3,4}$ Furthermore, the co-rotating twin-screw extruder can handle high viscosity substances as well as granular materials and performs chemical reactions without solvents. ${ }^{1,5}$ Then it can be considered as an intensified chemical reactor. 
As far as modeling of such processes is concerned, one has to consider highly coupled nonlinear phenomena involving highly viscous Newtonian or non-Newtonian fluid flows, heat transfer and chemical reactions. These processes occur within a complex non-stationary volume delimited by the barrel and the rotating screws. Another point is that the fluid viscosity can significantly change due to composition and temperature modifications.

The main point for the modeling of extrusion processes is the flow modeling. Two approaches are described in the literature. ${ }^{1}$ The first one consists in solving the $3 \mathrm{D}$ momentum balance equations, the second one is based on a simplified description of the flows by a network of ideal elementary flow models. However, in practice, the published works can only be roughly classified according to these categories since many models that have been proposed are the result of a combination of these two approaches.

The classical view of chemical reaction engineering consists in representing the flow through an extruder by a network of ideal elementary flow models like CSTR, Plug Flow with or without axial dispersion and so on. The parameters of these flow models have to be estimated from inert tracer experiments performed at a given steady state: this is the main drawback of this approach that is not predictive, the estimated parameters being valid only for the operating conditions under consideration. ${ }^{1,6,7,8}$ However, some insights on the TSE behavior can be gained from the analysis of experimental RTDs. A regular behavior can be observed for a given screw configuration that is mainly linked to the specific flow rate $\frac{Q}{N}$ where $Q$ is the melt volumetric flow rate and $N$ the screw rotation speed. ${ }^{9,10,11}$ By using an emulsifier as a tracer, the mixing performances of a TSE that is used for polymer blending are obtained by measuring the so-called emulsification curve. ${ }^{12}$

A global flow model can be derived by the combination of a detailed picture of the flow based on local solutions of the momentum balance equation and a network of ideal reactors. Using such an approach leads to predictive models with respect to the scale that is adopted. The momentum balance equation under consideration is generally a 1D linear model leading to local expressions of the melt flow rate so that one can deal with piecewise variations of the melt properties along the screw. By assuming or calculating the length of the partially filled and fully filled regions, the global RTD is predicted ${ }^{13-15}$ and the conversion and temperature profiles is calculated by using the steady state energy and material balances. ${ }^{16-18}$

When only 1D models are used to derive a global model of a TSE, no dispersion is present and the system is equivalent to a Plug Flow Reactor. Such 1D models are derived from the analytical or numerical solutions of 2D models leading to the computation of the melt flow rate, the axial pressure and filling ratio profiles. ${ }^{19,20}$ The dispersion that is due to the velocity profile can be included in the $1 \mathrm{D}$ models by using spatial averaging techniques ${ }^{21}$ or from velocity profiles. ${ }^{22}$

As far as dynamic modeling is concerned, many studies are based on 1D models. The global flow is equivalent to PFRs in series representing fully filled and partially filled zones, the boundary between these zones moving according to the operating conditions. ${ }^{23}$ Such models have been used to simulate the start-up of extruders. ${ }^{24,25}$ The possibility of multiple steadystate of the fully filled-partially filled boundary in the case of isothermal flows ${ }^{26}$ as well as the occurrence of oscillating behaviors ${ }^{27,28}$ have been pointed out. 
As far as our work is concerned with global dynamic modeling, we do not provide here any literature analysis devoted to 3D models. ${ }^{29-32}$ As far as we know, the main difficulties of these models are the non-stationary feature of the flow due to the screw rotation and the occurrence of free surface flows in the partially filled zones in combination with confined flows in the fully filled zones. Consequently, only a part of the extruder is generally represented. For example, mixing capacities of kneading disks have been recently studied by numerical simulations and local RTD measurements. ${ }^{33}$ Some references concerning the 3D approach are also available in the Vergnes and Berzin state of the art. ${ }^{1}$

In this paper, we adapt the model that we have previously developed for reactive extrusion processes dynamic modeling and control $^{34,35}$ to simulate the finishing step of Nylon-66 polycondensation operated in a co-rotating twin-screw extruder by the Rhodia company.,4 The main purpose of this finishing step is to perform water degassing of the polymer. In order to experimentally validate our model, we use transient responses of a TSE small-scale pilot plant to the injection of a tracer made of glass fibers. The paper is organized as follows. After a description of the main feature of our model, we provide some simulations to qualitatively illustrate its capabilities to reproduce the dynamic behavior of a TSE. The results concerning the degassing vent position validation with respect to the operating conditions are provided illustrating the capabilities of our model to be used at steady state for design purposes. Finally, we describe the glass fibers injection transient experiments that have been performed to validate our approach by comparing the experimental and simulated responses.

\section{Description of the dynamic model}

The model that we have previously developped ${ }^{34,35}$ is based on the combined approach of fluid flow modeling and chemical reaction engineering as referred in the Introduction section. The global flow is considered as a cascade of CSTRs with possible backflows. The upstream and downstream melt flow rates connecting these CSTRs are calculated by using a local 1D model depending on the screw configuration and melt properties. Our model has been primarily developed for control purposes so that it includes a description of the thermal dynamic behavior of the barrel. The corresponding input is then the heat flow within the barrel instead of the barrel temperature set point. The other inputs of the model are the inlet flow rate and composition, the screw rotation speed, the degassing pressure at the vent position. Subject to variations of these inputs, the transient behavior of the TSE can be simulated: filling ratio, pressure, temperature, composition profiles transients within the melt, behavior of the melt at the die outlet as well as temperature profile transients within the barrel. From long time simulations and constant inputs, the steady state behavior of the process is also obtained. Let us note that a steady state version of our model has been recently applied to the modeling of a TSE used for biopolymers oxidation. ${ }^{36}$

\subsection{Model equations}

Our model is based on a serial combination of basic elements similar to the one represented in Figure $1 . M_{i}(t)$, the time varying melt mass contained by the $i^{\text {th }}$ CSTR, is the solution of the following mass balance equation: 


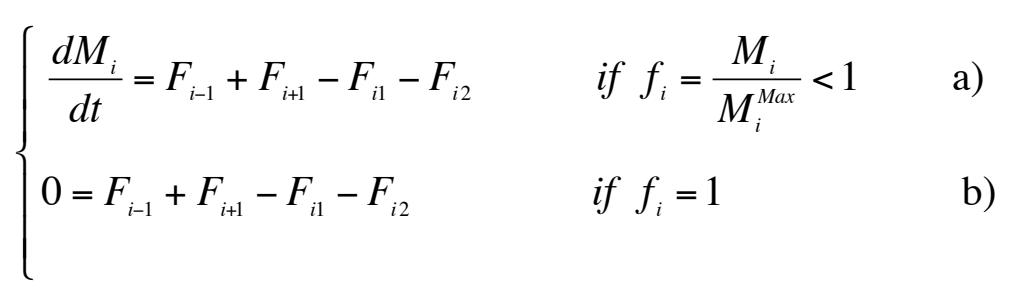

If $V_{i}^{M a x}=\frac{M_{i}^{M a x}}{\rho_{i}^{m}}$, the free volume of the $i^{\text {th }} \mathrm{CSTR}$, is full of melt, that is to say the filling ratio $f_{i}$ is equal to 1 , the corresponding steady state mass balance Equation (1b) gives a relation between the inlet and outlet mass flow rates that allows the computation of the pressure profile in the melt. Otherwise, the unsteady state mass balance Equation (1a) allows the computation of the time variation of $M_{i}=\rho_{i}^{m} f_{i} V_{i}^{M a x}$. Each $i^{\text {th }}$ CSTR is representative of a finite volume of melt the axial position of which being related to a piece of screw element. The downstream and upstream mass flow rates $F_{i 1}$ and $F_{i 2}$ (see Figure 1) are calculated according to the fact that the $i^{\text {th }}$ CSTR is associated to a direct, reverse screw element or a kneading block. The latter is considered to be equivalent to a direct or reverse screw element according to the staggering angle. ${ }^{19}$ Let us firstly consider that the $i^{\text {th }}$ CSTR represented in Figure 1 is associated to a piece of a direct screw element.

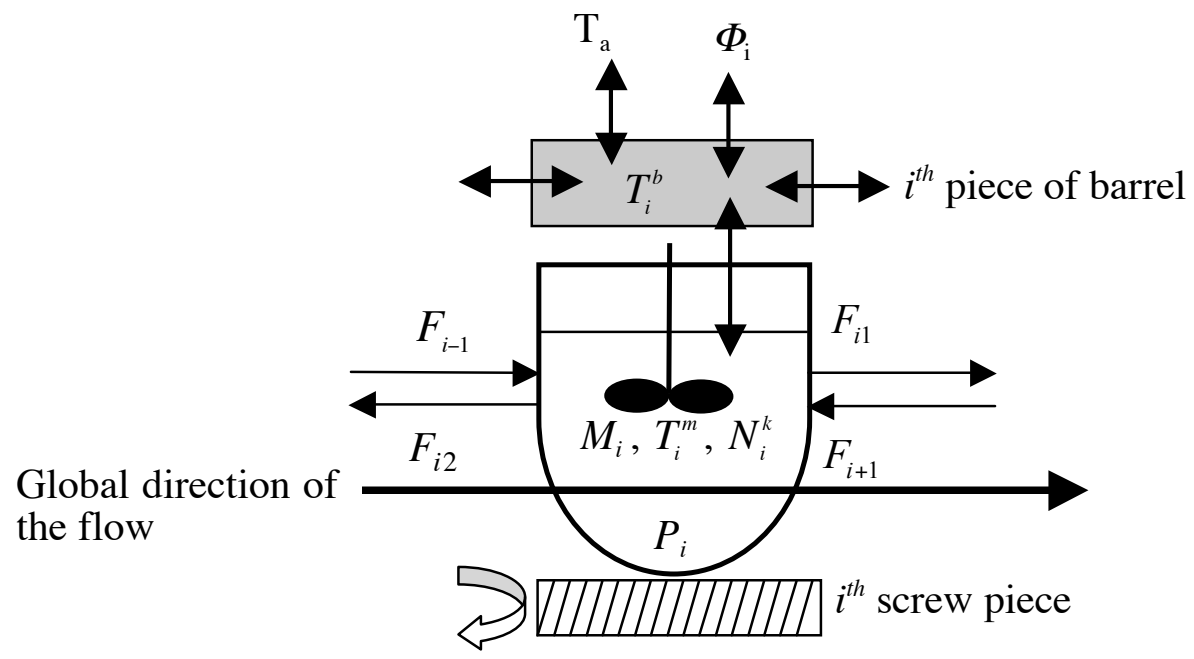

Figure 1: basic element for the global model building

The downstream mass flow rate $F_{i 1}$ is thus the drag flow corresponding to the pumping capacity of the screw element according to the filling ratio $f_{i}$. If $f_{i}<1$, the upstream mass flow rate $F_{i 2}$ is equal to zero since the pressure is constant and equal to the atmospheric pressure or to the pressure imposed by a possible degassing vent. On the contrary, a pressureinduced upstream flow $F_{i 2}$ is generated once $f_{i}=1$. If the $i^{\text {th }}$ CSTR represented in Figure 1 is associated to a piece of a reverse screw element, $F_{i 2}$ becomes the drag flow while $F_{i 1}$ becomes the pressure induced flow. The mass flow rates $F_{i-1}$ and $F_{i+1}$ depend on the nature of the screw elements associated with the $(i-1)^{\text {th }}$ and $(i+1)^{\text {th }}$ CSTRs as well as their filling ratio or pressure. The last $I^{\text {th }}$ CSTR of the cascade is the one situated just before the die so that $F_{I+1}=0$. If $f_{I}=1, F_{I 1}=F_{d i e}$. If $f_{I}<1, F_{I 1}=F_{I 2}=0$ since no pressure gradient is built at the die boundaries. Finally, at the junction between a direct and a reverse screw element, it turns to be necessary to insert a Poiseuille flow in order the melt flow to cross the reverse screw element. $^{37}$ 
The expressions of the flow rates are obtained by considering a simplified 1D local model that has been extensively used in the literature devoted to global TSE modeling. ${ }^{13-26,38}$ As far as the drag mass flow rate as well as the pressure flow can be upstream or downstream according to the screw configuration, the following expressions are used:

Direct screw:

Drag flow : $F_{i 1}=\rho_{i}^{m} K_{i d} \Omega f_{i} V_{i}^{M a x}$

Pressure flow : $F_{i 2}=\frac{K_{i p}\left(P_{i}-P_{i-1}\right)}{\eta_{i}^{m}}$

Reverse screw:

Drag flow : $F_{i 2}=\rho_{i}^{m} K_{i d} \Omega f_{i} V_{i}^{\text {Max }}$

Pressure flow : $F_{i 1}=\frac{K_{i p}\left(P_{i}-P_{i+1}\right)}{\eta_{i}^{m}}$

The die mass flow rate is classically expressed by the Poiseuille law:

$$
F_{\text {die }}=\frac{K_{d i e}\left(P_{I}-P_{0}\right)}{\eta_{I}^{m}}
$$

The parameters $K_{i d}, K_{i p}, K_{\text {die }}$ depend on the screw geometry ${ }^{38}$ while the melt viscosity $\eta_{i}^{m}$ is calculated according to the melt state within the $i^{\text {th }}$ CSTR. As far as the drag flow rate expression is concerned, it depends on the screw rotation speed so that a change in this input acts immediately all along the screw axis. This is the reason why an instantaneous peak of pressure is observed after a step change on the rotation speed..$^{34,35,39}$ Otherwise, the drag flow rate expression allows the computation of the filling process of the partially filled zones backward from restrictive elements. ${ }^{23-27}$ The dependence of the pressure-induced backflow with respect to the pressure gradient allows the computation of the pressure gradient within the fully filled zones. The pressure profile is calculated as follows. When the $i^{\text {th }}$ CSTR is fully filled, the steady state mass balance Equation (1b) is considered while if it is partially filled, its pressure is equal to the gaseous phase pressure $P_{i, 0}$ (the atmospheric pressure or the pressure imposed by a degassing vent). By considering simultaneously all these equations for all the CSTRs of the cascade, a linear system of equations is derived:

$$
\boldsymbol{A} \cdot \boldsymbol{P}=\boldsymbol{B}
$$

where $\boldsymbol{A}$ is a tridiagonal matrix having a 1 value on each diagonal position corresponding to a partially filled CSTR. $\boldsymbol{P}^{\mathrm{T}}=\left(\begin{array}{lllll}P_{1} & P_{2} & . & P_{I}\end{array}\right)$ is the pressure vector and $\boldsymbol{B}$ is a vector depending on known pressures and flow rates. It has been shown that the matrix $\boldsymbol{A}$ is $\operatorname{regular}^{34,35}$ so that it can be inverted to calculate $\boldsymbol{P}$ from Equation (5).

A very important point is that our model simulates the filling process of the TSE from the empty state or from any other set of initial conditions. The steady state filling ratio profile is then obtained for constant inputs and for long times without specifying in advance the position of the fully filled or partially filled zones. 
In order to calculate the other state variables of the melt flow, we use the material and energy balances associated to each CSTR. Let us assume that one chemical reaction $\sum_{k} v^{k} B^{k}=0$ having a molar rate defined with respect to the total mass $r^{m}$, occurs within the extruder. The material balance of the component $k$ in the $i^{\text {th }}$ CSTR is (see Figure 1):

$$
\frac{d N_{i}^{k}}{d t}=F_{i-1} c_{i-1}^{k}+F_{i+1} c_{i+1}^{k}-F_{i 1} c_{i}^{k}-F_{i 2} c_{i}^{k}+v^{k} r^{m}\left(T_{i}^{m}, c_{i}^{k}\right) M_{i}
$$

where $N_{i}^{k}$ is the mole number of component $k$ in the $i^{\text {th }}$ CSTR and $c_{i}^{k}=\frac{N_{i}^{k}}{M_{i}}$ the corresponding molar concentration per mass unit. Similarly, the energy balance for the melt contained in the $i^{\text {th }}$ CSTR is:

$$
\begin{aligned}
& M_{i} c_{p}^{m} \frac{d T_{i}^{m}}{d t}=F_{i-1} c_{p}^{m}\left(T_{i-1}^{m}-T_{i}^{m}\right)+F_{i+1} c_{p}^{m}\left(T_{i+1}^{m}-T_{i}^{m}\right)+f_{i} \alpha_{m} S_{m}\left(T_{i}^{b}-T_{i}^{m}\right) \\
& +f_{i} K_{d i s} \eta_{i}^{m} \Omega^{2}+M_{i} r^{m}\left(T_{i}^{m}, c_{i}^{k}\right)\left[-\Delta_{r} H\right]
\end{aligned}
$$

In this paper, we have neglected the influence of the screw mass on the thermal behavior of the $\mathrm{TSE}^{34,35}$ with respect to the one of the barrel. A piece of barrel is associated to the $t^{t h} \mathrm{CSTR}$ (see Figure 1) of which the temperature $T_{i}^{b}$ is assumed to be uniform. The corresponding energy balance is:

$$
\begin{aligned}
& M_{i}^{b} c_{p}^{b} \frac{d T_{i}^{b}}{d t}=\alpha_{a} S_{a}\left(T_{a}-T_{i}^{b}\right)+\alpha_{m} S_{m} f_{i}\left(T_{i}^{m}-T_{i}^{b}\right) \\
& +\frac{\lambda_{b} S_{b}}{e_{i}}\left(T_{i-1}^{b}-T_{i}^{b}\right)+\frac{\lambda_{b} S_{b}}{e_{i-1}}\left(T_{i+1}^{b}-T_{i}^{b}\right)+\Phi_{i}
\end{aligned}
$$

Axial heat transfer as well as heat transfer with the melt and the ambient air are considered. $\Phi_{i} \leq 0$ or $\Phi_{i} \geq 0$ is the heat flow that is generated by the regulation system through the thermal equipment of the barrel.

In this model, the melt properties - ie - the viscosity $\eta^{m}$, the mass density $\rho^{m}$, the chemical reaction rate $r^{m}$ - are considered to vary with the state of the melt. In the case of a nonNewtonian behavior of the melt, a local mean value of the shear rate can be calculated to compute $\eta_{i}^{m} \cdot 34,35$

In order to represent the degassing step, we have introduced the corresponding water flux in the water material balance of the CSTRs that are in relation with the degassing vent:

$$
J_{i}^{W}=\beta_{i}^{W}\left(c_{i}^{W}-c_{i}^{W e q}\left(T_{i}^{m}, P_{i, 0}\right)\right)
$$

In Equation (9), we have assumed that the degassing process occurs at the melt-gaseous phase interface ${ }^{3,40}, c_{i}^{W \ell q}$ being the melt equilibrium water concentration corresponding to the vent pressure $P_{i, 0}$.

2.2 Dynamic behavior of the model: some simulation examples 
In this section, we provide some dynamic simulations to illustrate the behavior of the model. The reaction under consideration is the Nylon-66 synthesis process operated in a TSE for the final degassing step. To this end, a degassing vent is used to impose a low pressure at a given position of the extruder. This position is chosen in order the filling ratio corresponding to the operating point to be less than one so that the degassing process can safely occur. The polycondensation of Nylon-66 is a reaction between an amine (A) and a carboxyl (C) end groups to form an amide linkage (L) with water elimination (W):

$$
A+C \leftrightarrow L+W
$$

The following reaction rate has been used for this polymerization reaction:

$$
r_{p}^{m}=\rho^{m} C_{T} k_{p}\left(x_{A} x_{C}-\frac{x_{L} x_{W}}{K_{e q}}\right)
$$

It should be noted that the polymer synthesis is always accompanied by some degradation reactions that can alter its end-use properties. We have considered one step of degradation:

$$
C \rightarrow S E+W
$$

where $S E$ stands for cyclized end group. The rate equation for this degradation reaction is as follows:

$$
r_{d}^{m}=\rho^{m} C_{T} k_{d} x_{C}
$$

The rate constants $k_{p}$ and $k_{d}$ and the equilibrium constant $K_{e q}$ expressions involved in the rate expressions (11) and (13) can be found in literature. ${ }^{4}$

As far as the melt viscosity varies during the polymer synthesis due to temperature and composition variations, we have used an empirical viscosity equation that is also available in the open literature ${ }^{41}$ :

$$
\begin{aligned}
& I V=0.036\left(\frac{2000}{C_{A}+C_{C}}\right)^{0.85} \\
& \eta^{m}\left(T_{\text {Ref }}\right)=0.026(I V)^{2.3}
\end{aligned}
$$

where $I V$ is the so-called viscosity index. In order to illustrate the role of viscosity in the coupling between the flow and the temperature profile in the melt, we have included a decreasing function with temperature for the melt viscosity calculation:

$$
\begin{aligned}
& \eta^{m}(T)=\eta^{m}\left(T_{R e f}\right) e^{\frac{E}{R}\left(\frac{1}{T}-\frac{1}{T_{R e}}\right)} \\
& T_{\text {Ref }}=573 \mathrm{~K} \\
& \frac{E}{R}=10000 \mathrm{~K}
\end{aligned}
$$


The screw of the TSE that is simulated is $1200 \mathrm{~mm}$ length, its external diameter is $40 \mathrm{~mm}$. It is represented by a cascade of 22 CSTRs: the CSTRs 7 and 15 correspond to inverse elements while the other ones are direct elements.

One can see in Figures 2 and 3 the time responses of the TSE with respect to two step changes of the feed flow rate $F_{0}$. In Figure 2 is represented the die pressure response. The final steady state pressure is lower due to the global decrease of the flow rate.

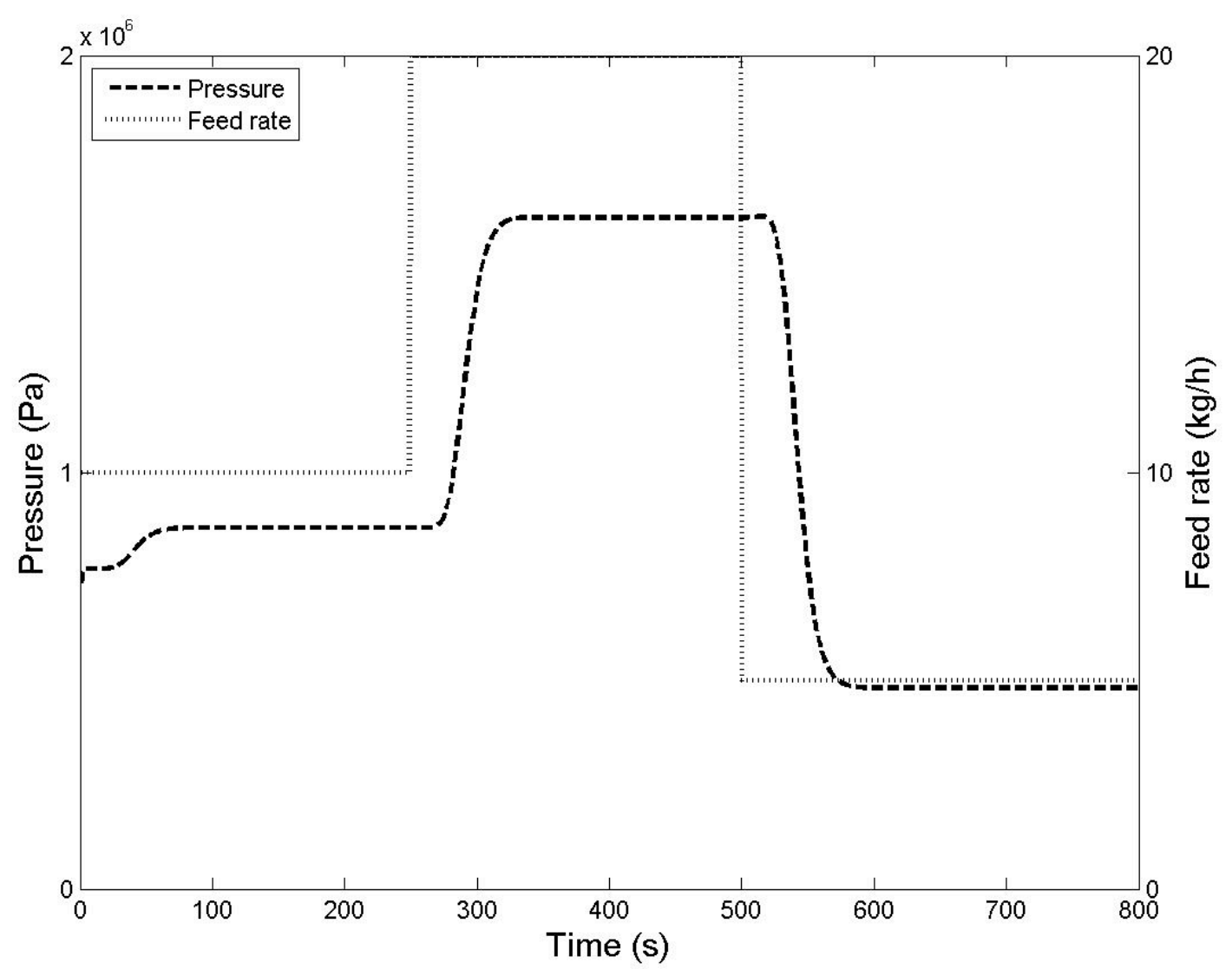

Figure 2: die pressure response to step changes of the feed flow rate for $N=200 \mathrm{rpm}$

In Figure 3 are represented the evolution of the filling ratio of some CSTRs of the cascade. The filling ratio of the $6^{\text {th }}$ CSTR that is situated upstream the $7^{\text {th }}$ inverse element is almost already full before the first positive step change on $F_{0}$. The tendency of CSTRs 14, 19 and 21 is also to fill after the positive step on $F_{0}$ since they are respectively situated upstream the $15^{\text {th }}$ inverse element and the die. Such a dynamic behavior of the boundaries between fully filled and partially filled zones has been described in the case of the start-up process of extruders $\mathrm{s}^{24,25}$ where the filling process is initiated from the die and continues by the backward propagation of the point where the fill ratio becomes equal to one. Conversely, for a negative step change of $F_{0}$, the tendency of the filling ratio of the CSTRs under consideration is to decrease as it can be seen in Figure 3. 


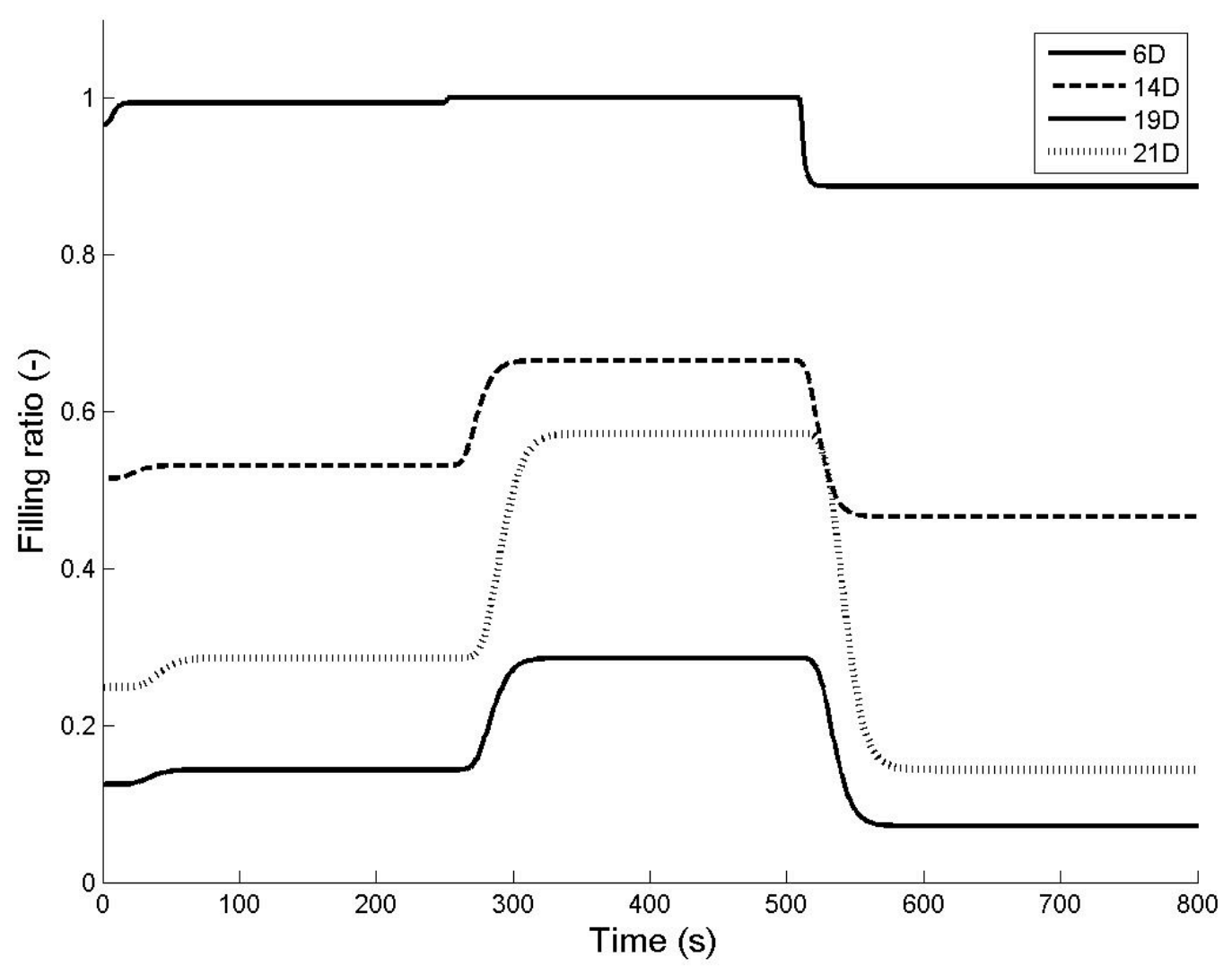

Figure 3: filling ratio response to step changes of the feed flow for $N=200 \mathrm{rpm}$

One can see in Figure 4 the time response of the die pressure $P_{I}$ with respect to two successive positive and negative screw rotation speed step changes. 


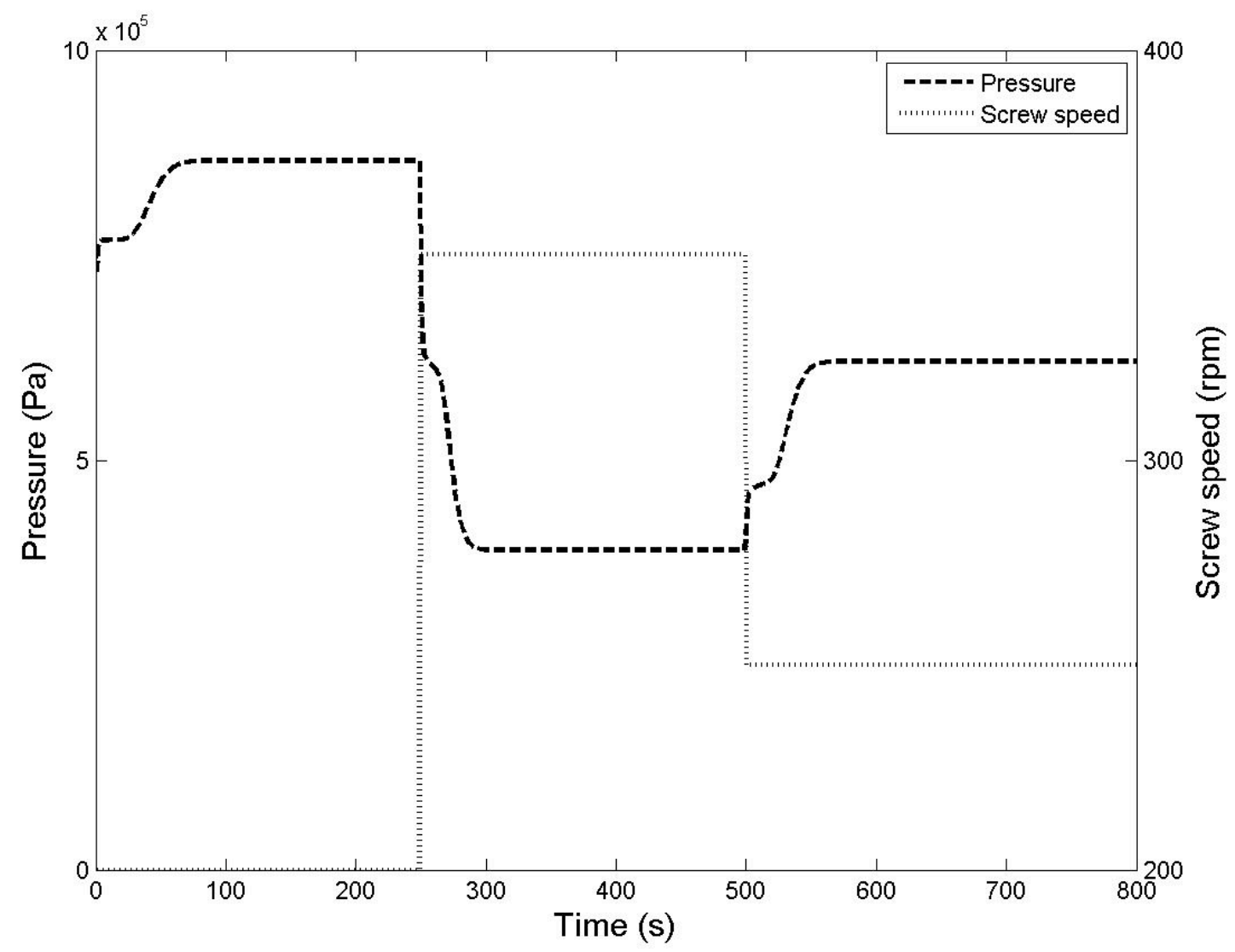

Figure 4: die pressure response with respect to step changes of the screw speed for

$$
F_{0}=10 \mathrm{~kg} \cdot \mathrm{h}^{-1}
$$

One can see the instantaneous responses of the pressure due to the direct dependence of the drag flow rate to the screw rotation speed (Equations (2) and (3)). Such a behavior has been observed experimentally. ${ }^{34,35,39}$ Then, the pressure tends to a lower or a higher value then the one prevailing before the rotation speed step changes according to the fact that it is positive or negative. This effect is due to the influence of the temperature on the viscosity: when the screw rotation speed increases, the viscous dissipation leads to an increase of the temperature as it can be seen in Figure 5. Consequently, a decrease of the viscosity and then a decrease of the pressure loss in the die occur. A symmetric behavior is observed when the screw rotation speed decreases. 


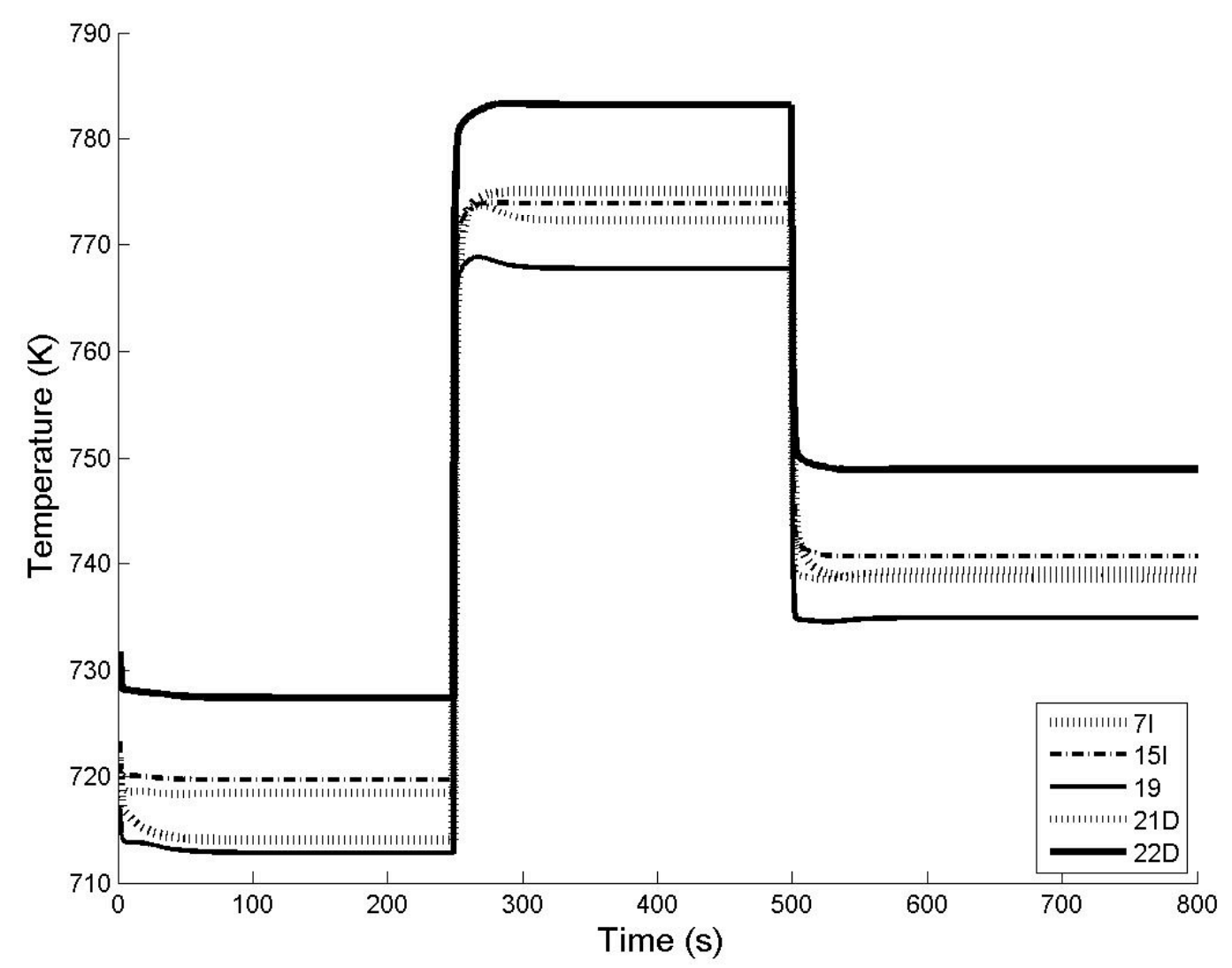

Figure 5: Temperature evolution in the melt contained in the CSTRs 7, 15, 19, 21, 22 after positive and negative step changes of the screw speed for $F_{0}=10 \mathrm{~kg} \cdot \mathrm{h}^{-1}$

Finally, in Figure 6 are represented three steady state filling ratio profiles corresponding to three screw rotation speeds.

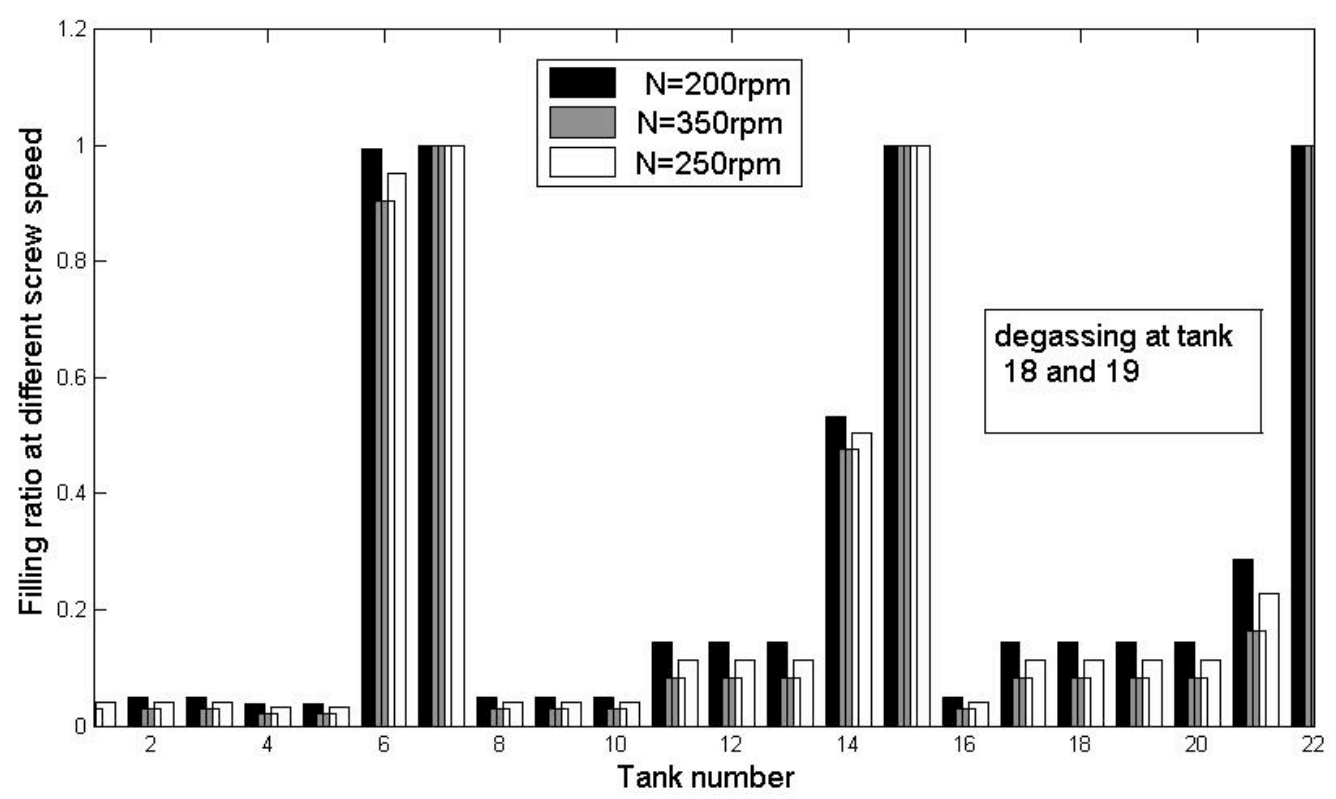

Figure 6: filling ratio profiles for $N=200,250$ and $350 \mathrm{rpm}$ and $F_{0}=10 \mathrm{~kg} \cdot \mathrm{h}^{-1}$ 
It can be seen that our model can be used for design purpose since the simulated steady state filling ratio profiles are compatible with the position of the degassing vent on the TSE pilot plant.

\section{Experimental results and model validation}

\subsection{Glass fibers injection transient experiments}

The aim of these experiments that are usually performed at the "Centre de Recherches et de Technologies de Lyon - CTRL" of the Rhodia Company is to get in situ information about the global flow through TSE processes. To this end, a TSE small-scale pilot plant is used. For confidentiality reason, we cannot provide a detailed description of the screw geometry and configuration as well as the TSE pilot plant operating conditions. The experiments principle is represented on Figure 7.

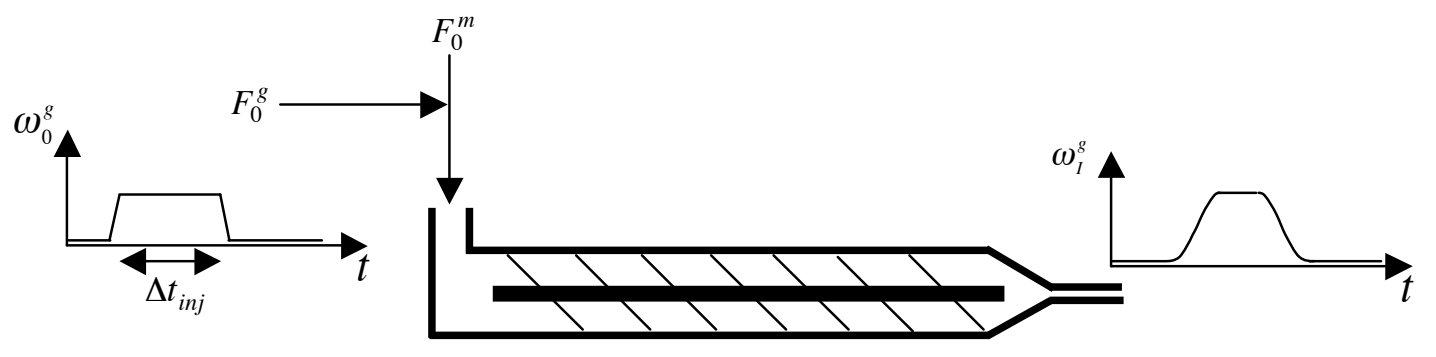

Figure 7: Principle of the glass fiber injection at the TSE inlet

As far as the Nylon-66 finishing and degassing step is concerned, the TSE is firstly operated at a given steady state in the TSE that is fed with a molten polymer flow $F_{0}^{m}$. At a given time that is taken as the origin, a mass flow rate of glass fibers $F_{0}^{g}$ is added to the inlet melt flow during the injection duration $\Delta t_{i n j}$. During the experiments, melt samples are taken at the TSE outlet and the glass fibers mass fraction is determined by calcinating the sample in order to determine the ash. Let us note that, on the one hand, this experiment cannot lead to the experimental RTD since, due to the high quantity of glass fibers that is injected, the melt density and viscosity are greatly modified so that the glass fibers are not an inert tracer from the flow point of view. On the other hand, from the chemical point of view, the glass fibers do not play any role. However, this nonlinear dynamic experiment can be used to check for the validity of our model provided that the glass fibers transport process description is included in the Nylon-66 finishing and degassing step model.

\subsection{Glass fibers transport modeling}

The glass fibers are considered as a supplementary non-reacting component of the melt. The mass density and the viscosity of the melt-glass fibers mixture are then expressed as a function of the glass fibers mass fraction $\omega^{g}$. The molten polymer-glass fibers mass density is given by the following expression:

$$
\rho^{m}\left(\omega^{g}\right)=\frac{1}{\frac{\omega^{g}}{\rho^{g}}+\frac{1-\omega^{g}}{\rho^{p}}}
$$


where $\rho^{g}$ and $\rho^{p}$ are respectively the mass densities of the glass and of the molten polymer. Since the glass fibers have a great influence on the mixture viscosity, we have used the following equation to estimate this viscosity that is suggested for concentrated suspensions of non-spherical particles ${ }^{42}$ :

$$
\eta^{m}\left(\phi^{g}\right)=\eta^{p}\left(T^{m}, c_{k}^{m}\right)\left(1-\frac{\phi^{g}}{\phi^{M a x}}\right)^{-A \phi^{M a x}}
$$

where $\phi^{g}$ is the glass fibers volumetric fraction. The parameters $A$ and $\phi^{\operatorname{Max}}$ are provided in Bird et $a l .^{42}$ for different type of particles. In our case, we have used cylindrical particles presenting an axial ratio of 7 .

\subsection{Comparison of experimental results with simulations}

Since the TSE response to glass fibers injection is measured during the Nylon-66 finishing step operation, the model also includes the representation of this process. To this end, we have used chemical rate $r^{m}\left(T^{m}, c_{k}^{m}\right)$ and molten polymer viscosity $\eta^{p}\left(T^{m}, c_{k}^{m}\right)$ expressions that have been provided by Rhodia Company: these expressions are not given here for confidentiality reasons.

One can see on Figures 8,9 and 10 a comparison between the simulated and the measured TSE outlet glass fibers mass fraction responses.

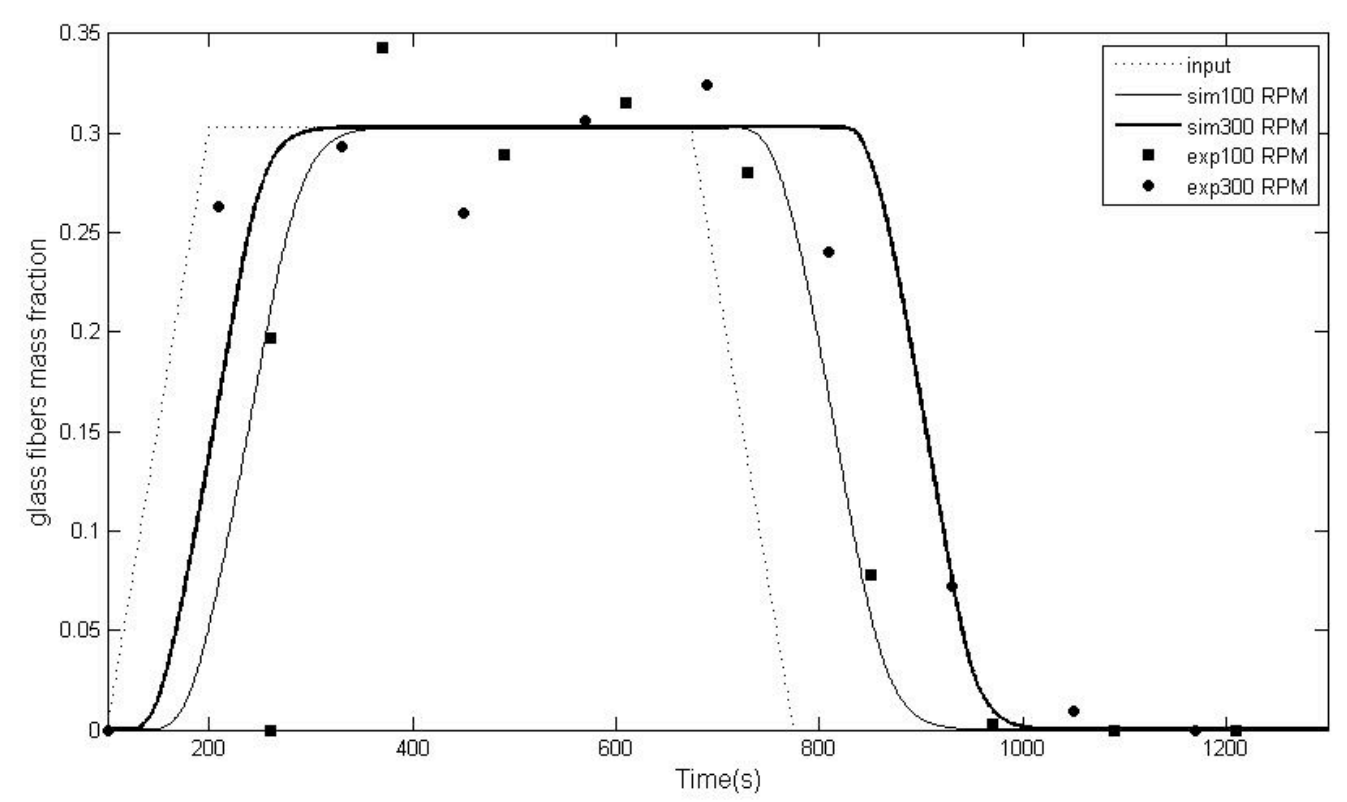

Figure 8: TSE response to a glass fibers injection for $N=100$ and $300 \mathrm{rpm}$ and $F_{0}^{m}=5 \mathrm{~kg} \cdot \mathrm{h}^{-1}$ 


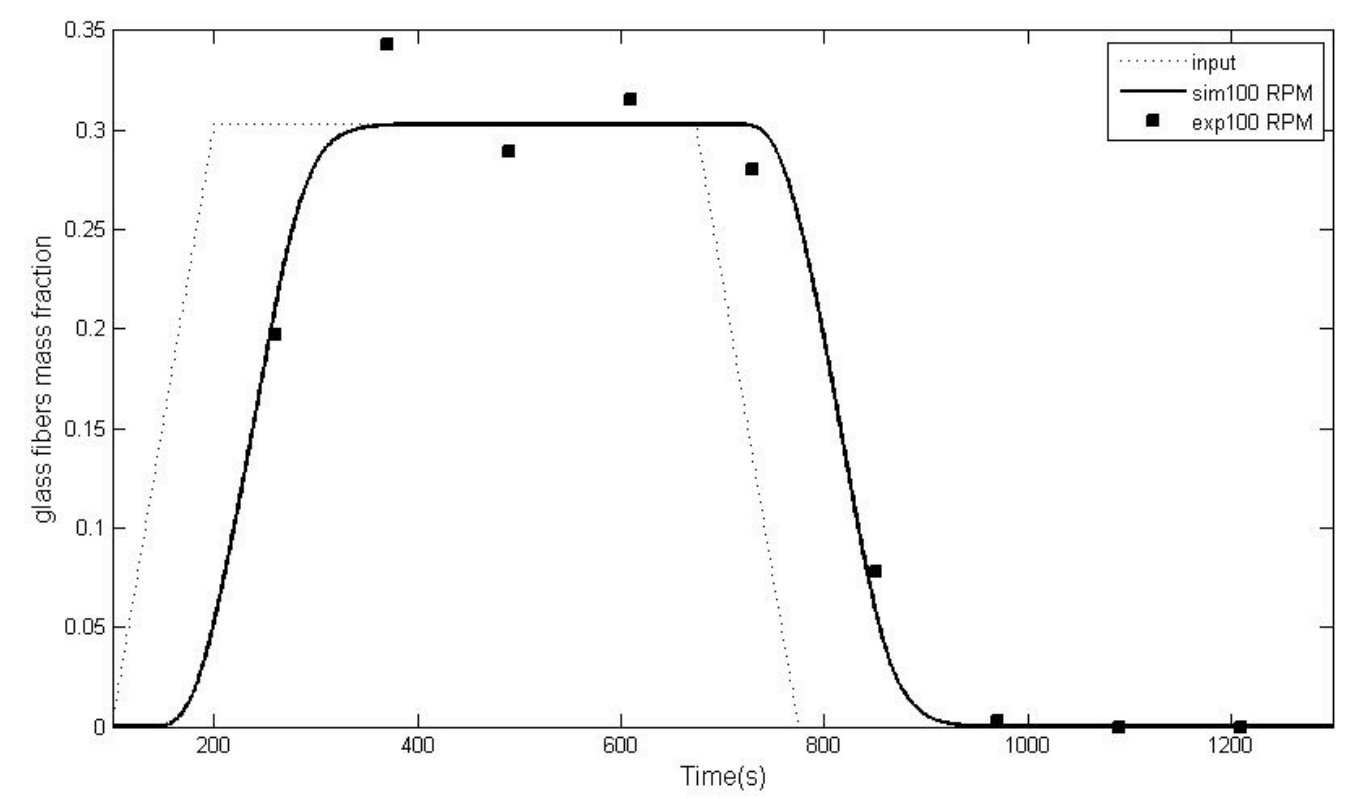

Figure 9: TSE response to a glass fibers injection for $N=100 \mathrm{rpm}$ and $F_{0}^{m}=15 \mathrm{~kg} \cdot \mathrm{h}^{-1}$

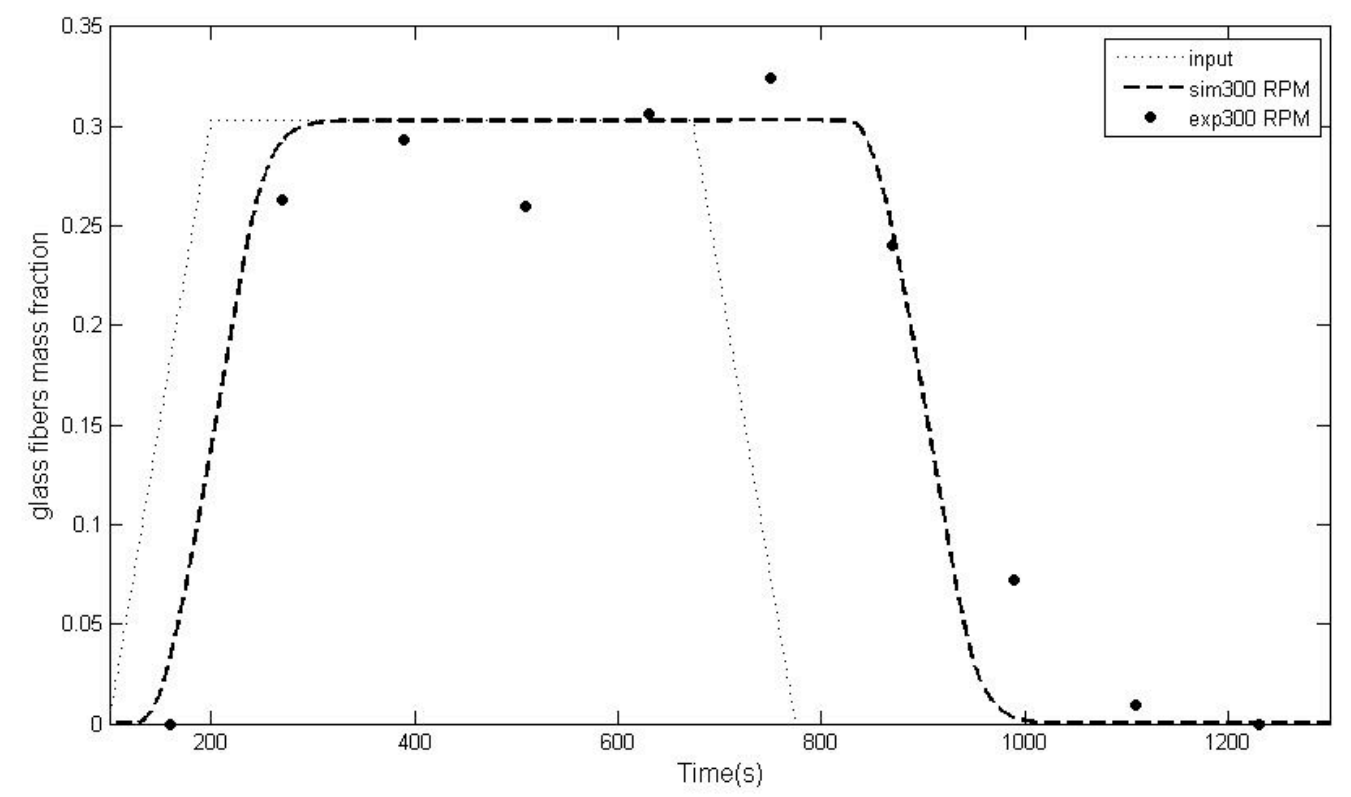

Figure 10: TSE response to a glass fibers injection for $N=300 \mathrm{rpm}$ and $F_{0}^{m}=15 \mathrm{~kg} \cdot \mathrm{h}^{-1}$

Our model satisfactorily fits the experimental behavior of the TSE. For this application, no parameter adjustment was necessary. This is the main point of our approach that can be totally predictive although the model is based on a network of ideal reactors and macroscopic balances. ${ }^{42}$ This is due to the fact that the rates of the flows connecting these ideal reactors are calculated from a local 1D flow model and that the volumes of these ideal reactors are deduced from the total available volume of the extruder that is known. For other applications $^{34,35}$, it was necessary to estimate some of the parameters of the models like $K_{d}$ and $K_{p}$ for example. This is due to the macroscopic scale that is adopted for this model that leads to high simplifications of the flow representation. However, this macroscopic scale is well adapted for the process control domain and is also the one that is used within the framework of very well known commercially available softwares for TSE design. ${ }^{1}$ 


\section{Conclusion}

In this paper, we have adapted a TSE dynamic model that we have previously developed for control purpose for the dynamic description of the degassing step of the Nylon-66 synthesis as it is performed in a TSE by the Rhodia Company. After a description of the model, we have provided some simulations to illustrate the capabilities of our model to simulate the TSE dynamic behavior. At steady state, our model reproduces filling ratio profiles that are compatible with the degassing vent position so that it can be used for design purposes. Responses to injection of glass fibers at the inlet of a TSE small-scale pilot plant operating the Nylon-66 finishing step have been obtained experimentally. Without any parameter estimation, the glass fibers transport through the TSE is satisfactorily reproduced thus validating the model.

Acknowledgment: thanks to «Axelera », the French competitive cluster «Chemistry and environment Lyon and Rhône-Alpes » for having supported this work within the working package « Process intensification ». 


\section{Literature cited}

(1) Vergnes, B.; Berzin, F. Modeling of reactive systems in twin-screw extrusion: challenges and applications. C. R. Chimie, 2006, 9, 1409-1418.

(2) Carneiro, O.S.; Caldeira, G.; Covas, J. A. Flow patterns in twin-screw extruders. Journal of Materials Processing Technology, 1999, 92-93, 309-315.

(3) Giudici, R.; Do Nascimento, C.A.O.; Capocchi Beiler, I.; Scherbakoff, N. Modeling of industrial Nylon-6,6 polycondensation process in a twin-screw extruder reactor. I. Phenomenological model and parameter adjusting. Journal of Applied Polymer Science, 1998, $67,1573-1587$.

(4) Giudici, R.; Do Nascimento, C.A.O.; Capocchi Beiler, I.; Scherbakoff, N. Transients experiments and mathematical modeling of an industrial twin-screw extruder reactor for Nylon-6,6 polymerization. Ind. Eng. Chem. Res., 1997, 36, 3513-3519.

(5) Hu, G.H.; Lambla, M. Fundamentals of reactive extrusion: an overview, in: Materials science and technology, a comprehensive treatment, ed. Cahn, R.W.; Haasen, P.; Kramer, E.J., vol 18: Processing of polymers, Wiley-VCH: Weinheim, 1997.

(6) Puaux, J.P.; Bozga, G.; Ainser, A. Residence time distribution in a corotating twin-screw extruder. Chemical Engineering Science, 2000, 55, 1641-1651.

(7) Puaux, J.P.; Cassagnau, P.; Bozga, G.; Nagy, I. Modelling of polyurethane synthesis by reactive extrusion. Chemical Engineering and Processing, 2006, 45, 481-487.

(8) Liu, H.Y.; Yao, Z.; Cao, K.; Li B.G. Characteristic analysis on a reactive extrusion process for the imidization of poly(styrene-co-maleic anhydride) with aniline. Chemical Engineering Science, 2010, 65, 1781-1789.

(9) Gao, J.; Walsh, G.C.; Biglio, D.; Briber, R.M.; Wetzel, M.D. Residence time distribution model for twin-screw extruders. AIChE J., 1999, 45, 2541-2549.

(10) Gao, J.; Walsh, G.C.; Biglio, D.; Briber, R.M.; Wetzel, M.D. Mean residence time analysis for twin screw extruders. Polymer Engineering and Science, 2000, 40, 227-237.

(11) Hu, G.H.; Kadir, I.; Picot, C. On-line measurement of the residence time distribution in screw extruders. Polymer Engineering and Science, 1999, 39, 930-939.

(12) Zhang C.L. ; Feng L.F. ; Hoppe S.; Hu G.H. Residence time distribution: an old concept in chemical engineering and a new application in polymer processing, AIChE Journal, 2009, $55,279-283$.

(13) de Graaf, R.A.; Rohde, M.; Janssen L.P.B.M. A novel model predicting the residencetime distribution during reactive extrusion. Chemical Engineering Science, 1997, 23, 43454356. 
(14) Poulesquen, A.; Vergnes, B. A study of residence time distribution in co-rotating twinscrew extruders. Part I: Theoretical modeling. Polymer Engineering and Science, 2003, 43, 1841-1848.

(15) Poulesquen, A.; Vergnes, B.; Cassagnau, P.; Michel, A.; Carneiro, O.S.; Covas, J.A.A study of residence time distribution in co-rotating twin-screw extruders. Part II: Experimental validation. Polymer Engineering and Science, 2003, 43, 1849-1862.

(16) Ganzeveld, K. J.; Capel, J. E.; Van der Wal, D. J.; Janssen, L.P.B.M. The modeling of a counter-rotating twin screw extruders as reactors for single-component reactions. Chemical Engineering Science, 1994, 49, 1639-1649.

(17) Michaeli, W.; Grefenstein, A.; Berghaus, U. Twin-screw extruders for reactive extrusion. Polymer Engineering and Science, 1995, 35, 1485-1504.

(18) Zagal, A.; Vivaldo-Lima, E.; Manero, O. A mathematical model for the reactive extrusion of methyl methacrylate in a co-rotating twin-screw extruder. Ind. Eng. Chem. Res., $2005,44,9805-9817$.

(19) Vergnes, B.; Della Valle, G.; Delamare, L. A global computer software for polymer flows in corotating twin screw extruders. Polymer Engineering and Science, 1998, 38, 17811792.

(20) Fukuoka, T. Numerical anlysis of a reactive extrusion process. Part I: kinetics study on grafting of vinylsilane to polyethylene. Part II: Simulations and verifications for the twin screw extrusion. Polymer Engineering and Science, 2000, 40, 2511-2538.

(21) Suresh, A.; Chakraborty, S.; Kargupta, K.; Ganguly, S. Low-dimensional models for describing mixing effects in reactive extrusion of polypropylene. Chemical Engineering Science, 2008, 63, 3788-3801.

(22) Chen, L.; Hu, G.H. Residence time distribution in screw extruders. AIChE J., 1993, 39, $1455-1464$.

(23) Diagne, M.; Dos Santos Martins, V.; Couenne, F.; Maschke, B.; Jallut C. Modélisation d'un procédé d'extrusion par deux systèmes d'équations d'évolution couplées par une interface. JESA (Journal Européen des Systèmes Automatisés), 2011, 45(7-10), 665 - 691.

(24) Kim, E.K.; White, J.L. Isothermal transient startup of starved flow modular co-rotating twin screw extruder. Polymer Engineering and Science, 2000, 40, 543-553.

(25) Kim, E.K.; White, J.L. Non-isothermal transient startup of starved flow modular corotating twin screw extruder. International Polymer Processing, 2000, 15, 233-241.

(26) Janssen, L.P.B.M.; Rozendal, P.F.; Hoogstraten, H.W.; Cioffi, M. A dynamic model for multiple steady states in reactive extrusion. International Polymer Processing, 2001, 16, 263271. 
(27) Janssen, L.P.B.M.; Rozendal, P.F.; Hoogstraten, H.W.; Cioffi, M. A dynamic model accounting for oscillating behavior in reactive extrusion. International Polymer Processing, $2003,18,277-284$.

(28) Mudalamane, R.; Bigio, D. I. Process variations and the transient behavior of extruders. AIChE J., 2003, 49, 3150-3160.

(29) Cheng, H.; Manas-Zloczower, I. Distributive mixing in conveying elements of a ZSK-53 co-rotating twin screw extruder. Polymer Engineering and Science, 1998, 38, 926-935.

(30) Funatsu, K.; Kihara, S.I.; Miyazaki, M.; Katsuki, S.; Kajiwara, T. 3-D numerical analysis on the mixing performance for assemblies with filled zone of right-handed and left-handed double-flighted screws and kneading blocks in twin-screw extruders. Polymer Engineering and Science, 2002, 42, 707-723.

(31) Zhu, L.; Narh, K.A.; Hyun, K.S. Evaluation of numerical simulation methods in reactive extrusion. Advances in Polymer Technology, 2005, 24, 183-193.

(32) Bertrand, F.; Thibault, F.; Delamare, L.; Tanguy, P.A. Adaptive finite element simulations of fluid flow in twin-screw extruders, Computers and Chemical Engineering, 2003, 27, 491-500.

(33) Zhang X.M. ; Feng L.F. ; Chen W.X. ; Hu G.H. Numerical simulation and experimental validation of mixing performance of kneading discs in a twin screw extruder, Polym. Eng. Sci., 2009, 49, 1772-1783.

(34) Choulak, S. Modeling and control of a reactive extrusion process (in french), $\mathrm{PhD}$ Thesis, Lyon University, 2004.

(35) Choulak, S.; Couenne, F.; Le Gorrec, Y.; Jallut, C.; Cassagnau, P.; Michel, A. Generic Dynamic Model for Simulation and Control of Reactive Extrusion. Ind. Eng. Chem. Res., 2004, 43, 7373-7382.

(36) de Ville d'Avray, M.A.; Isambert, A.; Brochot, S. Development of a global mathematical model for reactive extrusion processes in corotating twin-screw extruders. Computer-Aided Chemical Engineering, 2010, 28, 769-774.

(37) Agassant, J.F.; Avens, P.; Sergent, J.P.; Vergnes, B.; Vincent, M. La mise en forme des matières plastiques, Lavoisier, Tec et Doc: Paris, 1996.

(38) Booy, M.L. Isothermal flow of viscous liquids in corotating twin screw devices. Polymer Engineering and Science, 1980, 20, 1220-1228.

(39) Iqbal, M. H.; Sundararaj, U.; Shah, S.L. New approach to develop dynamic gray box model for a plasticating twin-screw extruder. Ind. Eng. Chem. Res., 2010, 49, 648-657.

(40) Gestring, I.; Mewes, D. Degassing of molten polymers. Chemical Engineering Science, 2002, 57, 3415-3426. 
(41) Giudici, R.; Nascimento, C.A.O.; Tresmondi, A.; Domingues, A.; Pellicciotta, R. Mathematical modeling of an industrial process of Nylon-6,6 polymerization in a two-phase flow tubular reactor. Chemical Engineering Science, 1999, 54, 3243-3449.

(42) Bird, R.B.; Stewart W.E.; Lightfoot E.N. Transport Phenomena, $2^{\text {Th }}$ edition, Wiley: New-York, 2002. 


\section{Nomenclature}

$A$ : regular matrix used to calculate the pressure profile

$B$ : chemical symbol

$\boldsymbol{B}$ : vector used to calculate the pressure profile

$c_{p}$ : constant pressure heat capacity

$c$ : molar concentration per mass unit

$C:$ molar concentration per volume unit

$e$ : barrel length between two elements

$E$ : activation energy

$F:$ melt mass flow rate

$f:$ fill ratio

$I$ : total number of CSTRs

$J$ : molar flux

$k$ : reaction rate constant

$K$ : model parameter

$M$ : melt mass

$N$ : mole number

$N$ : rotation speed

$P$ : pressure

$P$ : pressure vector

$(\mathrm{Pa})$

$Q$ : melt volumetric flow rate

$\left(\mathrm{m}^{3} \cdot \mathrm{s}^{-1}\right)$

$r$ : chemical reaction rate with respect to the melt mass

$\left(\mathrm{mol} . \mathrm{s}^{-1} \cdot \mathrm{kg}^{-1}\right)$

$R$ : ideal gas constant

$\left(\mathrm{J} \cdot \mathrm{mol}^{-1} \cdot \mathrm{K}^{-1}\right)$

$S$ : surface

$T:$ temperature

$t:$ time

$V:$ volume

$I V$ : viscosity index

\section{Greek symbols}

$\lambda$ : heat conductivity

$\alpha$ : heat transfer coefficient

$\beta$ : mass transfer coefficient

$\Phi$ : heat flow

$\phi$ : volumetric fraction

$\eta$ : dynamic viscosity

$\Delta_{r} H$ : reaction enthalpy

$\left(\mathrm{W} \cdot \mathrm{m}^{-1} \cdot \mathrm{K}^{-1}\right)$

$\left(\mathrm{W} \cdot \mathrm{m}^{-1} \cdot \mathrm{K}^{-1}\right)$

$\left(\mathrm{kg} \cdot \mathrm{m}^{-1} \cdot \mathrm{s}^{-1}\right)$

$\Omega$ : screw rotation speed

$\omega$ : mass fraction

$v:$ stoichiometric coefficient

$\rho$ : mass density

\section{Subscripts}

0 : vent or ambient pressure or inlet

1: downstream 


\author{
2: upstream \\ $A$ : amine end group \\ $C$ : carboxyl end group \\ $b$ : barrel \\ $d$ : drag flow or degradation reaction \\ die: related to the die \\ eq: equilibrium \\ $i: i^{\text {th }}$ CSTR \\ inj: injection \\ $L$ : amide linkage \\ $m$ : melt \\ Max: maximum value \\ $p$ : pressure flow or polymerization reaction \\ $S E$ : stabilized or cyclized end group \\ $T$ : total \\ $W$ : water \\ Superscripts \\ $a$ : ambient air \\ $b$ : barrel \\ dis: dissipation \\ eq: equilibrium value \\ $g$ : glass fibers \\ $k: k$ component \\ $m$ : melt \\ $p$ : molten polymer \\ $W$ : water \\ Max: maximum value
}

